\title{
Boll Retention (\%) under Different Environments/Sowing Conditions in Upland Cotton (Gossypium hirsutum L.)
}

\author{
Pinki", S.S. Siwach, R.S. Sangwan, Sombir Singh, V.S. Mor, Shiwani Mandhania, \\ Sunayana and Neha Rohila
}

CCS Haryana Agricultural University, Hisar, Haryana, India

*Corresponding author

\section{A B S T R A C T}

Keywords

Cotton, Boll retention, Sowing period, Different environments

Article Info

Accepted:

10 April 2018

Available Online:

10 May 2018
The present study was conducted to find out the Boll retention (\%) under different environments/sowing quality in three varieties H 1098 - I, H 1300 and $\mathrm{H} 1316$ under six environments (three sowing periods i.e. early, normal and late sown conditions in year 2015 and 2016). Observations were recorded for total flower tagged and total boll retained in different tagging period. Tagging period stared from June end when there were sizable amount of flowers. In early sown condition per cent boll retention was higher (59.1) and also produced more number of flowers as compared to normal sown $(51.9 \%)$ and late sown conditions (58.7\%) conditions. So, early sown condition was best for maximum boll retention.

\section{Introduction}

Cotton is an important commercial crop of India. Upland cotton dominates the world's cotton fiber production accounting for approximately $90 \%$ of the total production (Malagouda et al., 2014). Different species and cultivars of plants respond differently to climatic factors depending basically on the growth stage. The influence of those factors is modified by other factors such as water or nitrogen stress. Other environmental factors vary simultaneously with climatic factors causing an association between individual climatic variables and plant responses, and, thus, it seems impossible to determine causeeffect relationships. There is limited information on the response of cotton plants to climatic factors.

Sowing time is the main factor affecting yield, and considered as key element to investigate the appropriate sowing period of crop cultivars under the particular agro climatic conditions. Sowing time linearly affects the seed cotton yield, because early maturing cultivars start flowering and boll formation well earlier as compared to late ones. Growth and yield contributing parameters including fibre quality traits are closely associated with environments 
favourable for higher yields. The yield of cotton is mostly associated with sowing dates as boll weight and formation of bolls which are inter linked with the yield (Mahmood-ulHassan et al., 2003).

However, by determining the relationship of climatic factors with flower and boll production and retention, the overall level of production can be possibly predicted. So, the present investigation was planned to find out the appropriate time period for maximum boll retention.

\section{Materials and Methods}

The experiment was conducted during kharif 2015 \& 2016 having three cultivars H 1098-I, $\mathrm{H} 1300$ and $\mathrm{H} 1316$ of upland cotton grown at CCS Haryana Agricultural University, Hisar in randomized block design replicated six times each in eight rows of $6 \mathrm{~m}$ length with a spacing of $67.5 \times 30 \mathrm{~cm}$. These varieties were grown in six environments that comprises of three sowings periods (Early: first fortnight of April, normal: first fortnight of May and late: end of May/early June) during the year 2015 and 2016 (Table 1).

Data was recorded as in all the three replications in every week flowers were tagged and number of effective bolls formed from these flowers was counted and the week in which maximum and minimum bolls developed was identified. The data on total flower tagged and total boll retained was recorded from tagging period i.e. June, 24 to till the last picking of the experimental plots.

\section{Results and Discussion}

In early sown condition there were 10 flower tagging weeks from $24^{\text {th }}$ June to $26^{\text {th }}$ of August. In all the three varieties total flower tagged, total boll retained and (\%) boll retention were different during this period. In the variety $\mathrm{H} 1098$ - I total flower tagged were 6720 , boll retained were 3665 with $54.5 \%$ boll retention was 54.5. In variety $\mathrm{H} 1300$ flowers tagged were 7078, boll retained were 4485 with $63.4 \%$ boll retention and in variety $\mathrm{H}$ 1316 total flower tagged were 7047, boll retained were 4461 with $59 \%$ boll retention.

In normal sown condition there were 8 tagging weeks from $8^{\text {th }}$ July to $26^{\text {th }}$ of August. In all the three varieties total flower tagged, total boll retained and (\%) boll retention were different for these 8 weeks. In the variety H 1098- I total flower tagged were 4435 , boll retained were 2456 with55.4\% boll retention. In variety $\mathrm{H}$ 1300 total flower tagged were 4449, boll retained were 2628 with $59.1 \%$ boll retention and in variety $\mathrm{H} 1316$ total flower tagged were 4564, boll retained were 2718 with $59.6 \%$ boll retention.

In late sown condition there were 8 tagging weeks from $8^{\text {th }}$ July to $26^{\text {th }}$ of August. In all the three varieties total flower tagged, total boll retained and \% boll retention were different for these 8 weeks. In the variety $\mathrm{H}$ 1098- I total flower tagged were 3720, boll retained were 2001 with53.8\% boll retention. In variety $\mathrm{H} 1300$ total flower tagged were 3876 , boll retained were 2373 with $61.2 \%$ boll retention and in variety $\mathrm{H} 1316$ total flower tagged were 3481 , boll retained were 2126 with $61.1 \%$ boll retention (Table $2-4$ ).

Figure 1, 2 and 3 showing total flower tagged, total boll retained and \% boll retention in early sown conditions, figure 4, 5 and 6 for normal and figure 7, 8 and 9 for late sown conditions.

Critical period for flowering and boll retention under different sown condition was presented in Table 5, 6 and 7. Total flower tagged, total boll retained and (\%) boll retention in different sowing condition were different. In early sown condition there were 10 tagging weeks from $24^{\text {th }}$ June to $26^{\text {th }}$ of August. 
Table.1 Sowing dates and different environments in 2015 and 2016

\begin{tabular}{|c|c|c|c|}
\hline & Environment & Date of Sowing & $\begin{array}{c}\text { Environment } \\
\text { Designation }\end{array}$ \\
\hline $\mathbf{2 0 1 5}$ & Sowing period & 10 April & $\mathrm{E}_{1}$ \\
\hline & Early & $15 \mathrm{May}$ & $\mathrm{E}_{2}$ \\
\hline \multirow{2}{*}{$\mathbf{2 0 1 6}$} & Normal & 5 June & $\mathrm{E}_{3}$ \\
& Late & 26 April & $\mathrm{E}_{4}$ \\
& Early & 5 May & $\mathrm{E}_{5}$ \\
\hline & Normal & 2 June & $\mathrm{E}_{6}$ \\
\hline
\end{tabular}

Table.2 Flower tags and boll retention of different varieties in early sown condition

\begin{tabular}{|c|c|c|c|c|c|c|c|c|c|c|}
\hline Early & & $\begin{array}{c}\mathrm{H} \\
1098-\mathrm{I}\end{array}$ & & & $\begin{array}{c}\mathrm{H} \\
1300\end{array}$ & & & $\begin{array}{c}\mathrm{H} \\
1316\end{array}$ & & \\
\hline $\begin{array}{c}\text { Tagging } \\
\text { week } \\
\end{array}$ & $\begin{array}{l}\text { Tagging } \\
\text { period }\end{array}$ & $\begin{array}{c}\text { Flower } \\
\text { tag }\end{array}$ & $\begin{array}{c}\text { Boll } \\
\text { retained }\end{array}$ & $\begin{array}{c}\% \text { boll } \\
\text { retention }\end{array}$ & $\begin{array}{c}\text { Flower } \\
\text { tag }\end{array}$ & $\begin{array}{c}\text { Boll } \\
\text { retained }\end{array}$ & $\begin{array}{c}\% \text { boll } \\
\text { retention }\end{array}$ & $\begin{array}{c}\text { Flower } \\
\text { tag }\end{array}$ & $\begin{array}{c}\text { Boll } \\
\text { retained }\end{array}$ & $\begin{array}{c}\% \text { boll } \\
\text { retention }\end{array}$ \\
\hline 1 & June 24- 30 & 727 & 274 & 37.7 & 833 & 487 & 58.5 & 855 & 429 & 50.2 \\
\hline 2 & July 1-7 & 767 & 391 & 51.0 & 865 & 498 & 57.6 & 803 & 515 & 64.1 \\
\hline 3 & July 8-14 & 705 & 407 & 57.7 & 630 & 394 & 62.5 & 765 & 469 & 61.3 \\
\hline 4 & July 15-21 & 617 & 344 & 55.8 & 714 & 422 & 59.1 & 652 & 350 & 53.7 \\
\hline 5 & July 22-28 & 473 & 263 & 55.6 & 579 & 380 & 65.6 & 852 & 490 & 57.5 \\
\hline 6 & July 29-4 & 617 & 381 & 61.8 & 919 & 588 & 64.0 & 751 & A479 & 63.8 \\
\hline 7 & Aug 5-11 & 787 & 410 & 52.1 & 835 & 529 & 63.4 & 607 & 363 & 59.8 \\
\hline 8 & Aug 12-18 & 630 & 377 & 59.8 & 569 & 401 & 70.5 & 679 & 414 & 61.0 \\
\hline 9 & Aug 19- 25 & 736 & 444 & 60.3 & 557 & 373 & 67.0 & 559 & 336 & 60.1 \\
\hline \multirow[t]{2}{*}{10} & Aug 26-1 sep & 661 & 374 & 56.6 & 577 & 413 & 71.6 & 524 & 316 & 60.3 \\
\hline & & 6720 & 3665 & 54.5 & 7078 & 4485 & 63.4 & 7047 & 4161 & 59.0 \\
\hline
\end{tabular}

Table.3 Flower tags and boll retention of different varieties in normal sown condition

\begin{tabular}{|c|c|c|c|c|c|c|c|c|c|c|}
\hline Normal & & $\begin{array}{c}H \\
1098\end{array}$ & & & $\begin{array}{c}H \\
1300\end{array}$ & & & $\begin{array}{c}\mathrm{H} \\
1316\end{array}$ & & \\
\hline $\begin{array}{c}\text { Tagging } \\
\text { week }\end{array}$ & $\begin{array}{l}\text { Tagging } \\
\text { period }\end{array}$ & $\begin{array}{c}\text { Flower } \\
\text { tag }\end{array}$ & $\begin{array}{c}\text { Boll } \\
\text { retained }\end{array}$ & $\begin{array}{c}\% \text { boll } \\
\text { retention }\end{array}$ & $\begin{array}{c}\text { Flower } \\
\text { tag }\end{array}$ & $\begin{array}{c}\text { Boll } \\
\text { retained }\end{array}$ & $\begin{array}{c}\% \text { boll } \\
\text { retention }\end{array}$ & $\begin{array}{c}\text { Flower } \\
\text { tag }\end{array}$ & $\begin{array}{c}\text { Boll } \\
\text { retained }\end{array}$ & $\begin{array}{l}\% \text { boll } \\
\text { retention }\end{array}$ \\
\hline 1 & July 8-14 & 516 & 292 & 56.6 & 517 & 293 & 56.7 & 640 & 355 & 55.5 \\
\hline 2 & July 15-21 & 477 & 274 & 57.4 & 584 & 364 & 62.3 & 511 & 275 & 53.8 \\
\hline 3 & July 22-28 & 493 & 270 & 54.8 & 532 & 301 & 56.6 & 611 & 375 & 61.4 \\
\hline 4 & July 29-4 & 590 & 304 & 51.5 & 719 & 438 & 60.9 & 690 & 374 & 54.2 \\
\hline 5 & Aug 5-11 & 654 & 386 & 59.0 & 604 & 356 & 58.9 & 516 & 306 & 59.3 \\
\hline 6 & Aug 12-18 & 539 & 270 & 50.1 & 461 & 282 & 61.2 & 547 & 366 & 66.9 \\
\hline 7 & Aug 19- 25 & 609 & 361 & 59.3 & 525 & 296 & 56.4 & 488 & 325 & 66.6 \\
\hline \multirow[t]{2}{*}{8} & Aug 26-1 sep & 557 & 299 & 53.7 & 507 & 298 & 58.8 & 561 & 342 & 61.0 \\
\hline & & 4435 & 2456 & 55.4 & 4449 & 2628 & 59.1 & 4564 & 2718 & 59.6 \\
\hline
\end{tabular}


Table.4 Flower tags and boll retention of different varieties in late sown condition

\begin{tabular}{|c|c|c|c|c|c|c|c|c|c|c|c|}
\hline $\begin{array}{c}\text { Late } \\
\text { Tagging } \\
\text { week }\end{array}$ & Tagging period & $\begin{array}{c}\text { Flower } \\
\text { tag }\end{array}$ & $\begin{array}{c}\text { Boll } \\
\text { retained }\end{array}$ & $\begin{array}{c}\text { \% boll } \\
\text { retention }\end{array}$ & $\begin{array}{c}\text { Flower } \\
\text { tag }\end{array}$ & $\begin{array}{c}\text { Boll } \\
\text { retained }\end{array}$ & $\begin{array}{c}\text { \% boll } \\
\text { retention }\end{array}$ & $\begin{array}{c}\text { Flower } \\
\text { tag }\end{array}$ & $\begin{array}{c}\text { Boll } \\
\text { retained }\end{array}$ & $\begin{array}{c}\text { \% boll } \\
\text { retention }\end{array}$ \\
\hline $\mathbf{1}$ & July 8-14 & 437 & 226 & 51.7 & 426 & 257 & 60.3 & 535 & 347 & 64.9 \\
\hline $\mathbf{2}$ & July 15-21 & 425 & 225 & 52.9 & 559 & 351 & 62.8 & 444 & 281 & 63.3 \\
\hline $\mathbf{3}$ & July 22-28 & 395 & 211 & 53.4 & 462 & 250 & 54.1 & 420 & 261 & 62.1 \\
\hline $\mathbf{4}$ & July 29-4 & 521 & 269 & 51.6 & 607 & 366 & 60.3 & 548 & 314 & 57.3 \\
\hline $\mathbf{5}$ & Aug 5-11 & 539 & 277 & 51.4 & 514 & 333 & 64.8 & 423 & 262 & 61.9 \\
\hline $\mathbf{6}$ & Aug 12-18 & 443 & 256 & 57.8 & 440 & 279 & 63.4 & 386 & 230 & 59.6 \\
\hline $\mathbf{7}$ & Aug 19- 25 & 537 & 288 & 53.6 & 434 & 272 & 62.7 & 393 & 229 & 58.3 \\
\hline $\mathbf{8}$ & Aug 26-1 sep & 423 & 249 & 58.9 & 434 & 265 & 61.1 & 332 & 202 & 60.8 \\
\hline & & 3720 & 2001 & 53.8 & 3876 & 2373 & 61.2 & 3481 & 2126 & 61.1 \\
\hline
\end{tabular}

Table.5 Critical period for flowering and boll retention under early sown condition

\begin{tabular}{|c|c|c|c|c|c|c|c|c|c|c|}
\hline Early & & \multicolumn{3}{|c|}{$\begin{array}{l}\text { Flower tagged in } \\
\text { different sowings }\end{array}$} & & \multicolumn{3}{|c|}{$\begin{array}{l}\text { No. of bolls } \\
\text { retained }\end{array}$} & \multirow[b]{2}{*}{ Total } & \multirow[b]{2}{*}{$\begin{array}{l}\% \text { boll } \\
\text { retention }\end{array}$} \\
\hline $\begin{array}{c}\text { Tagging } \\
\text { week } \\
\end{array}$ & $\begin{array}{l}\text { Tagging } \\
\text { period }\end{array}$ & $\begin{array}{c}H \\
1098\end{array}$ & $\begin{array}{c}\mathrm{H} \\
1300\end{array}$ & H1316 & Total & $\begin{array}{c}H \\
1098\end{array}$ & $\begin{array}{c}H \\
1300\end{array}$ & H1316 & & \\
\hline 1 & June 24- 30 & 727 & 833 & 855 & 2415 & 274 & 487 & 429 & 1190 & 49.3 \\
\hline 2 & July 1-7 & 767 & 865 & 803 & 2435 & 391 & 498 & 515 & 1404 & 57.7 \\
\hline 3 & July 8-14 & 705 & 630 & 765 & 2100 & 407 & 394 & 469 & 1270 & 60.5 \\
\hline 4 & July 15-21 & 617 & 714 & 692 & 2023 & 344 & 422 & 403 & 1169 & 57.8 \\
\hline 5 & July 22-28 & 473 & 579 & 652 & 1704 & 263 & 380 & 350 & 993 & 58.3 \\
\hline 6 & July 29-4 & 617 & 919 & 751 & 2287 & 381 & 588 & 479 & 1448 & 63.3 \\
\hline 7 & Aug 5-11 & 787 & 835 & 607 & 2229 & 410 & 529 & 363 & 1302 & 58.4 \\
\hline 8 & Aug 12-18 & 630 & 569 & 679 & 1878 & 377 & 401 & 414 & 1192 & 63.5 \\
\hline 9 & Aug 19- 25 & 736 & 557 & 559 & 1852 & 444 & 373 & 336 & 1153 & 62.3 \\
\hline 10 & Aug 26-1 sep & 661 & 577 & 524 & 1762 & 374 & 413 & 316 & 1103 & 62.6 \\
\hline & & 6720 & 7078 & 6887 & 20685 & 3665 & 4485 & 4074 & 12224 & 59.1 \\
\hline
\end{tabular}

Table.6 Critical period for flowering and boll retention under normal sown condition

\begin{tabular}{|c|l|c|c|c|c|c|c|c|c|c|}
\hline Normal & & \multicolumn{3}{|c|}{ Flower tagged in different } & & \multicolumn{3}{|c|}{ No. of bolls retained } & & \\
\hline $\begin{array}{c}\text { Tagging } \\
\text { week }\end{array}$ & Tagging period & H 1098 & H 1300 & H1316 & Total & H & H & H1316 & Total & $\begin{array}{c}\text { \% boll } \\
\text { retention }\end{array}$ \\
\hline $\mathbf{1}$ & July 8-14 & 516 & 630 & 765 & 1911 & 292 & 293 & 355 & 940 & 49.2 \\
\hline $\mathbf{2}$ & July 15-21 & 477 & 714 & 692 & 1883 & 274 & 364 & 374 & 1012 & 53.7 \\
\hline $\mathbf{3}$ & July 22-28 & 493 & 579 & 652 & 1724 & 270 & 301 & 275 & 846 & 49.1 \\
\hline $\mathbf{4}$ & July 29-4 & 590 & 919 & 751 & 2260 & 304 & 438 & 374 & 1116 & 49.4 \\
\hline $\mathbf{5}$ & Aug 5-11 & 654 & 835 & 607 & 2096 & 386 & 356 & 306 & 1048 & 50.0 \\
\hline $\mathbf{6}$ & Aug 12-18 & 539 & 569 & 679 & 1787 & 270 & 282 & 366 & 918 & 51.4 \\
\hline $\mathbf{7}$ & Aug 19- 25 & 609 & 557 & 559 & 1725 & 361 & 296 & 325 & 982 & 56.9 \\
\hline $\mathbf{8}$ & Aug 26-1 sep & 557 & 577 & 524 & 1658 & 299 & 298 & 342 & 939 & 56.6 \\
\hline
\end{tabular}


Table.7 Critical period for flowering and boll retention under late sown condition

\begin{tabular}{|c|c|c|c|c|c|c|c|c|c|c|}
\hline \multirow{2}{*}{$\begin{array}{c}\text { Late } \\
\text { Tagging } \\
\text { week } \\
\end{array}$} & \multirow[b]{2}{*}{$\begin{array}{l}\text { Tagging } \\
\text { period }\end{array}$} & \multicolumn{3}{|c|}{$\begin{array}{l}\text { Flower tagged in } \\
\text { different sowings }\end{array}$} & \multirow[b]{2}{*}{ Total } & \multicolumn{3}{|c|}{$\begin{array}{l}\text { No. of bolls } \\
\text { retained }\end{array}$} & \multirow[b]{2}{*}{ Total } & \multirow[b]{2}{*}{$\begin{array}{c}\% \text { boll } \\
\text { retention }\end{array}$} \\
\hline & & $\begin{array}{c}\text { H } \\
1098\end{array}$ & $\begin{array}{c}\mathrm{H} \\
1300\end{array}$ & H1316 & & $\begin{array}{c}\text { H } \\
1098\end{array}$ & $\begin{array}{c}\mathrm{H} \\
1300\end{array}$ & H1316 & & \\
\hline 1 & July 8-14 & 437 & 426 & 535 & 1398 & 226 & 257 & 347 & 830 & 59.4 \\
\hline 2 & July 15-21 & 425 & 559 & 444 & 1428 & 225 & 351 & 281 & 857 & 60.0 \\
\hline 3 & July 22-28 & 395 & 462 & 420 & 1277 & 211 & 250 & 261 & 722 & 56.5 \\
\hline 4 & July 29-4 & 521 & 607 & 548 & 1676 & 269 & 366 & 314 & 949 & 56.6 \\
\hline 5 & Aug 5-11 & 539 & 514 & 423 & 1476 & 277 & 333 & 262 & 872 & 59.1 \\
\hline 6 & Aug 12-18 & 443 & 440 & 386 & 1269 & 256 & 279 & 230 & 765 & 60.3 \\
\hline 7 & Aug 19- 25 & 537 & 434 & 393 & 1364 & 288 & 272 & 229 & 789 & 57.8 \\
\hline 8 & Aug 26-1 sep & 423 & 434 & 332 & 1189 & 249 & 265 & 202 & 716 & 60.2 \\
\hline & & 3720 & 3876 & 3481 & 11077 & 2001 & 2373 & 2126 & 6500 & 58.7 \\
\hline
\end{tabular}

Table.8 Boll retention \% in early, normal and late sown conditions

\begin{tabular}{|c|c|c|c|c|c|c|c|c|c|}
\hline & \multicolumn{4}{|c|}{ Total flower tagged } & \multicolumn{4}{|c|}{ Total boll retained } & $\%$ boll retention \\
\hline & $\begin{array}{c}\text { H } \\
1098\end{array}$ & $\begin{array}{c}\text { H } \\
1300\end{array}$ & $\begin{array}{c}H \\
1316\end{array}$ & Total & $\begin{array}{c}\text { H } \\
1098\end{array}$ & $\begin{array}{c}H \\
1300\end{array}$ & $\begin{array}{c}\text { H } \\
1316\end{array}$ & Total & $\%$ boll retention \\
\hline Early & 6720 & 7078 & 6887 & 20685 & 3665 & 4485 & 4074 & 12224 & 59.1 \\
\hline Normal & 4435 & 5380 & 5229 & 15044 & 2456 & 2628 & 2717 & 7801 & 51.9 \\
\hline Late & 3720 & 3876 & 3481 & 11077 & 2001 & 2373 & 2126 & 6500 & 58.7 \\
\hline Total & 14875 & 16334 & 15597 & 46806 & 8122 & 9486 & 8917 & 26525 & 56.56667 \\
\hline
\end{tabular}

Fig.1 Boll retention in H 1098-I in early sown condition

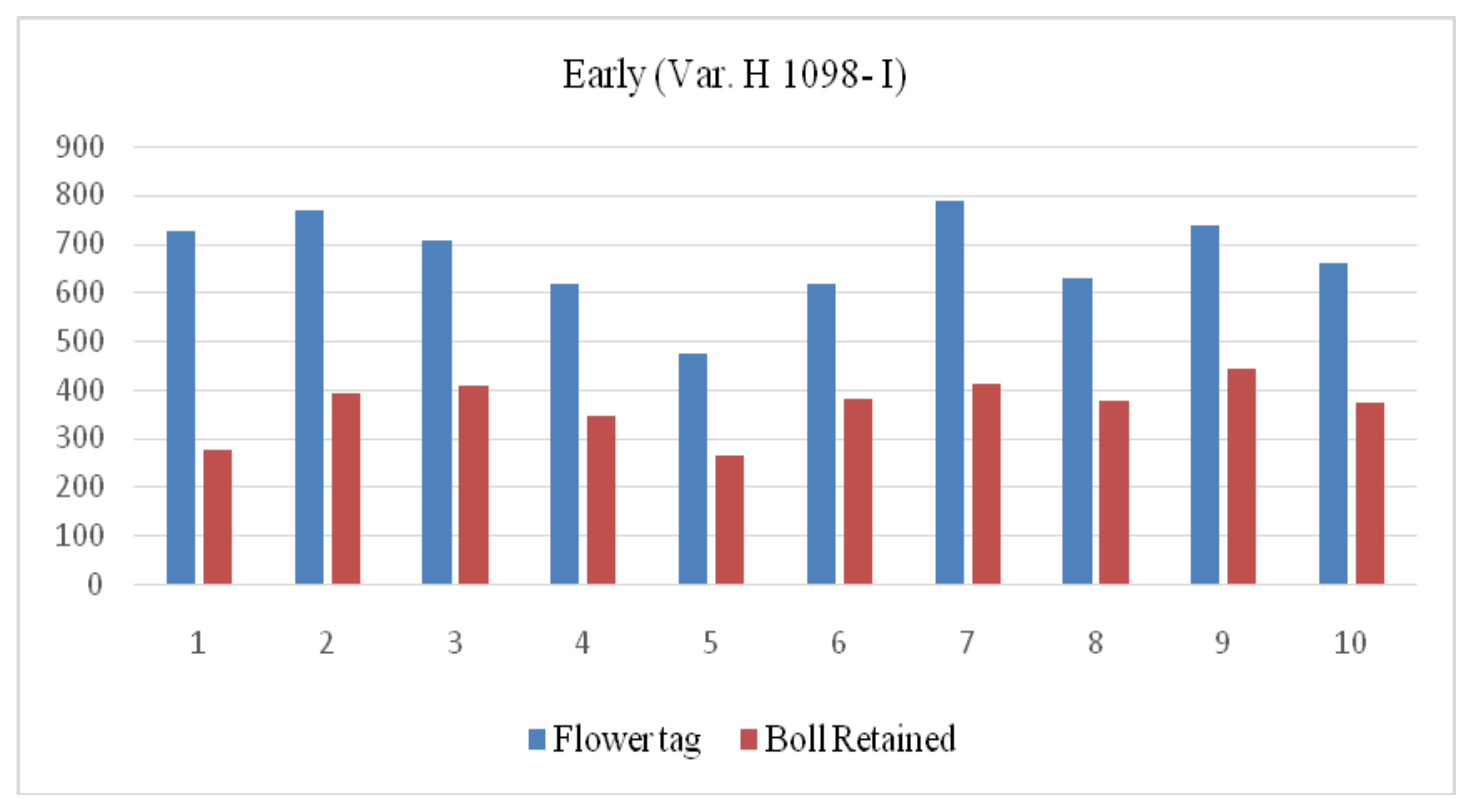


Fig.2 Boll retention in H 1300 in early sown condition

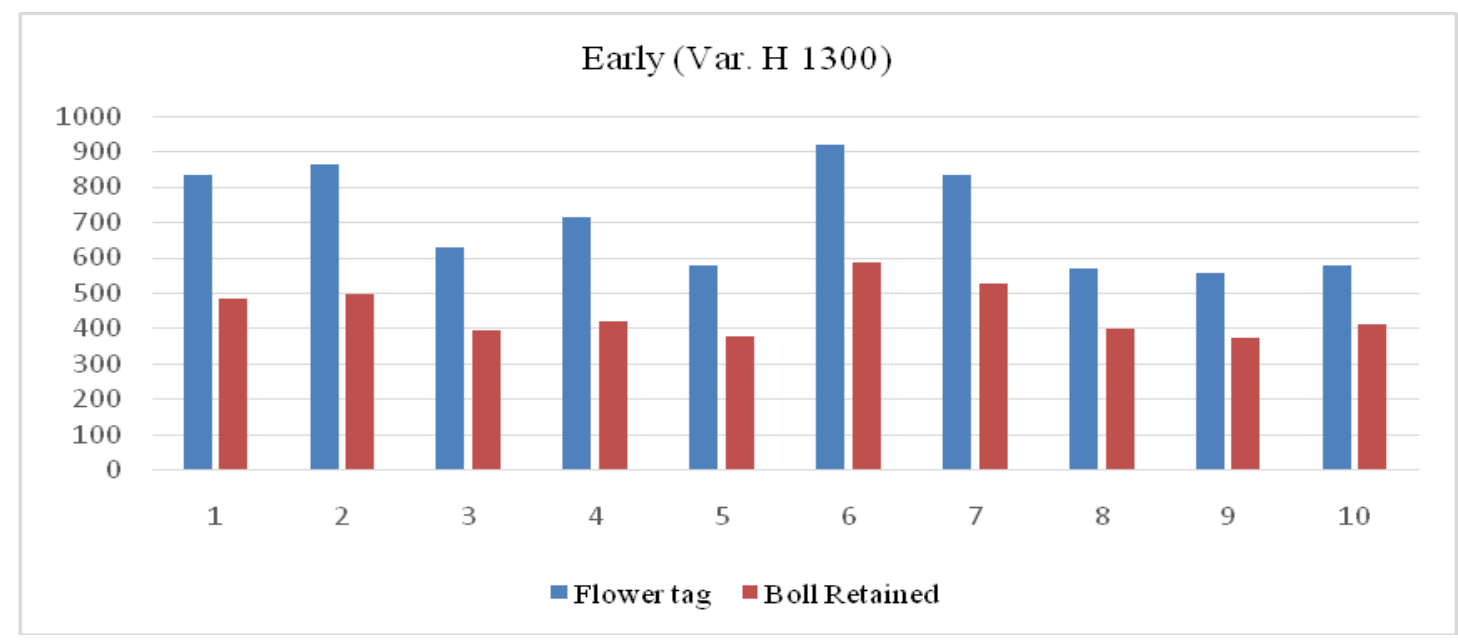

Fig.3 Boll retention in H 1316 in early sown condition

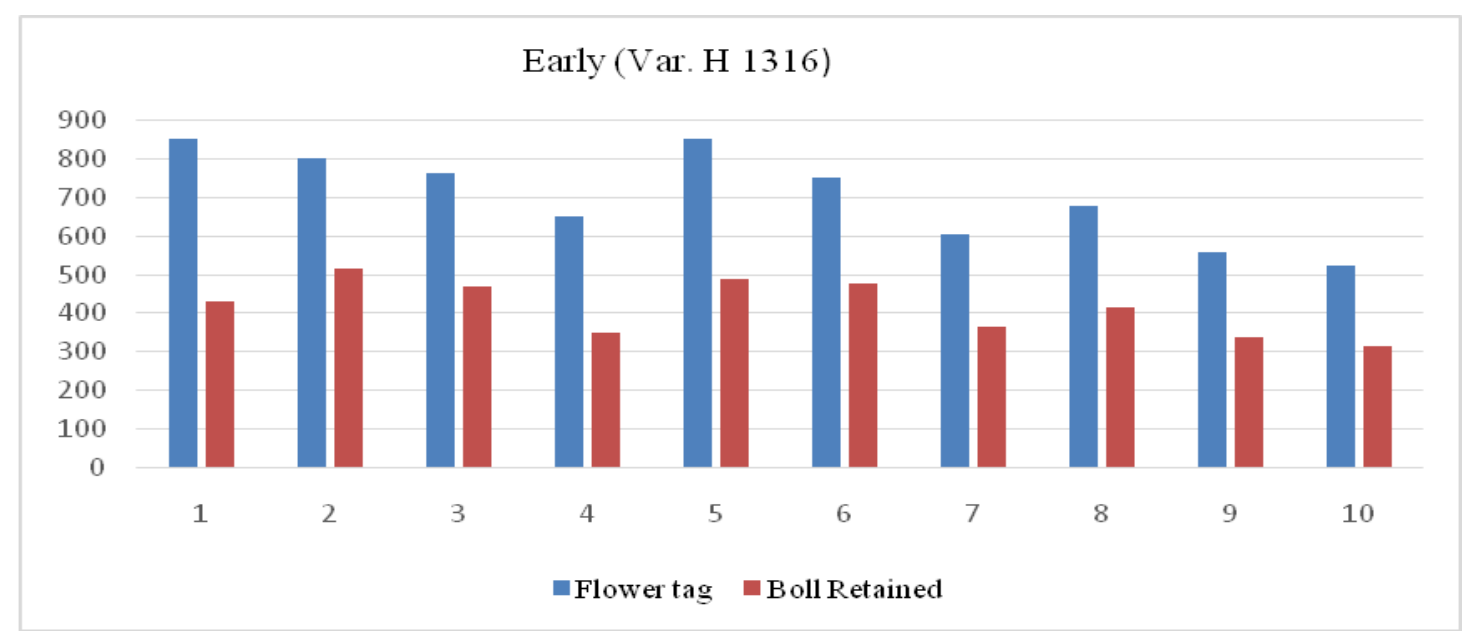

Fig.4 Boll retention in H1098 - I in normal sown condition

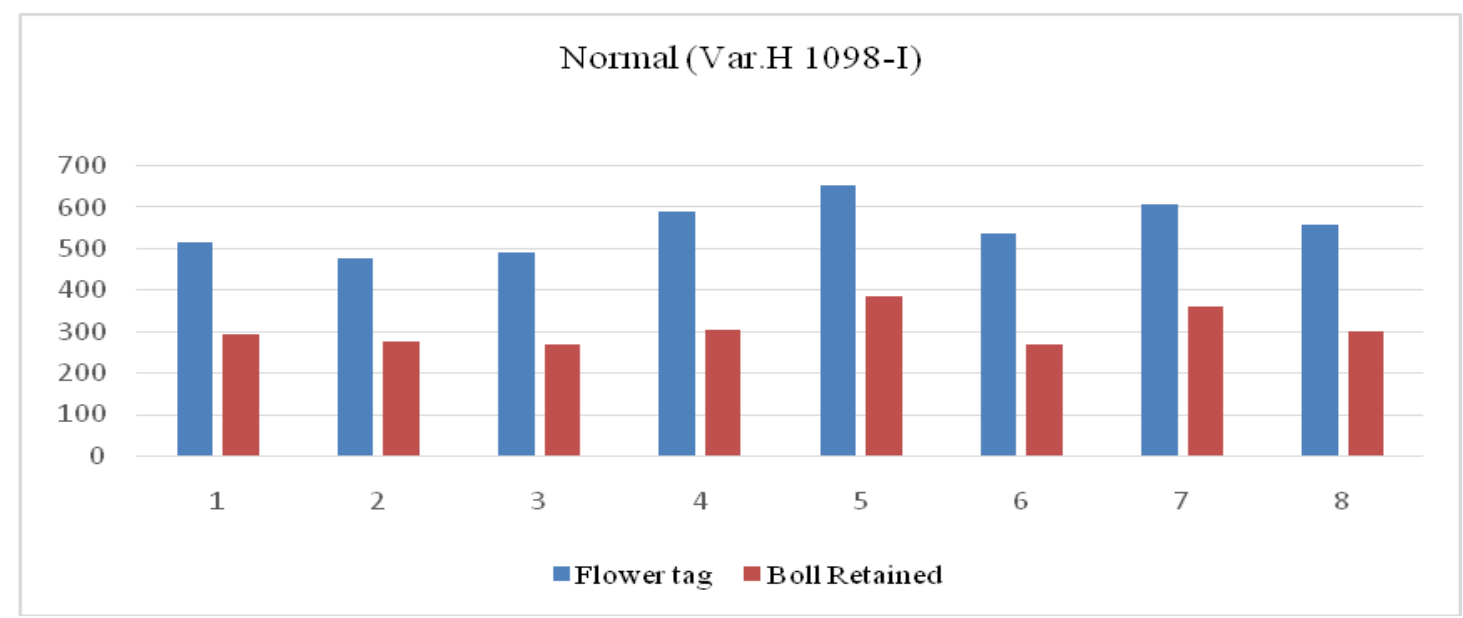


Fig.5 Boll retention in H 1300 in normal sown condition

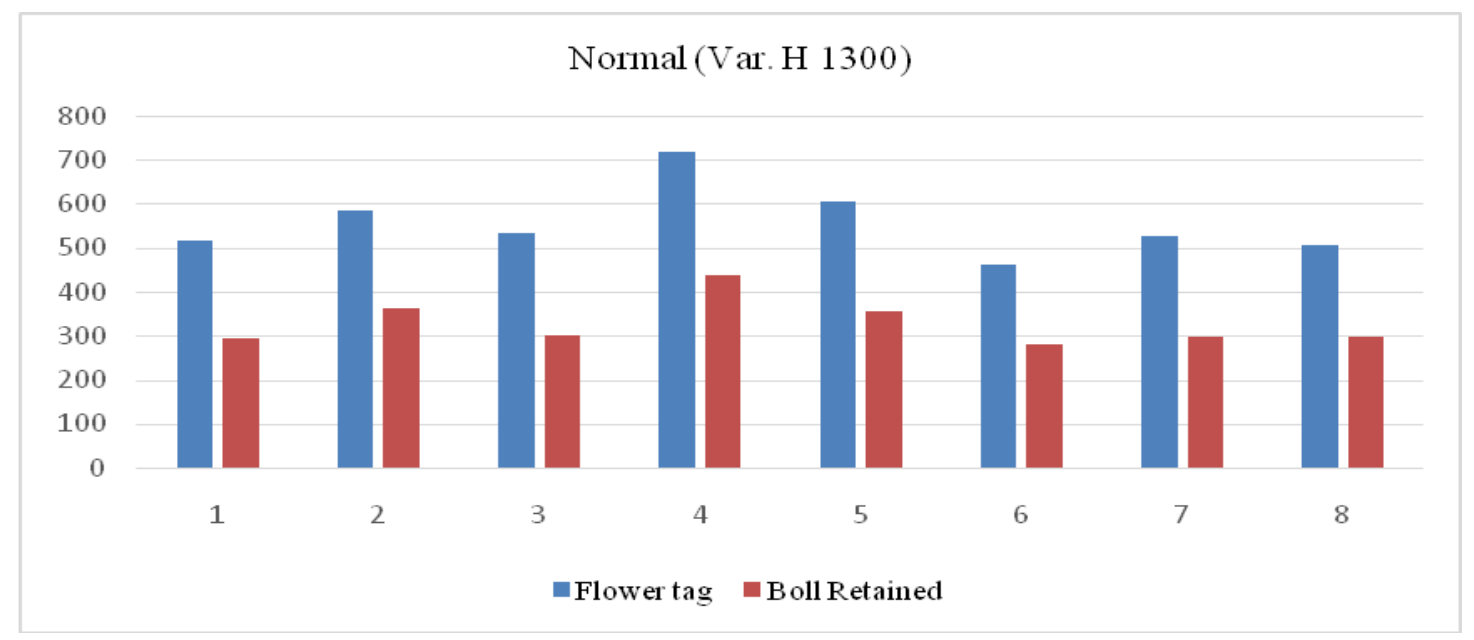

Fig.6 Boll retention in H 1316 in normal sown condition

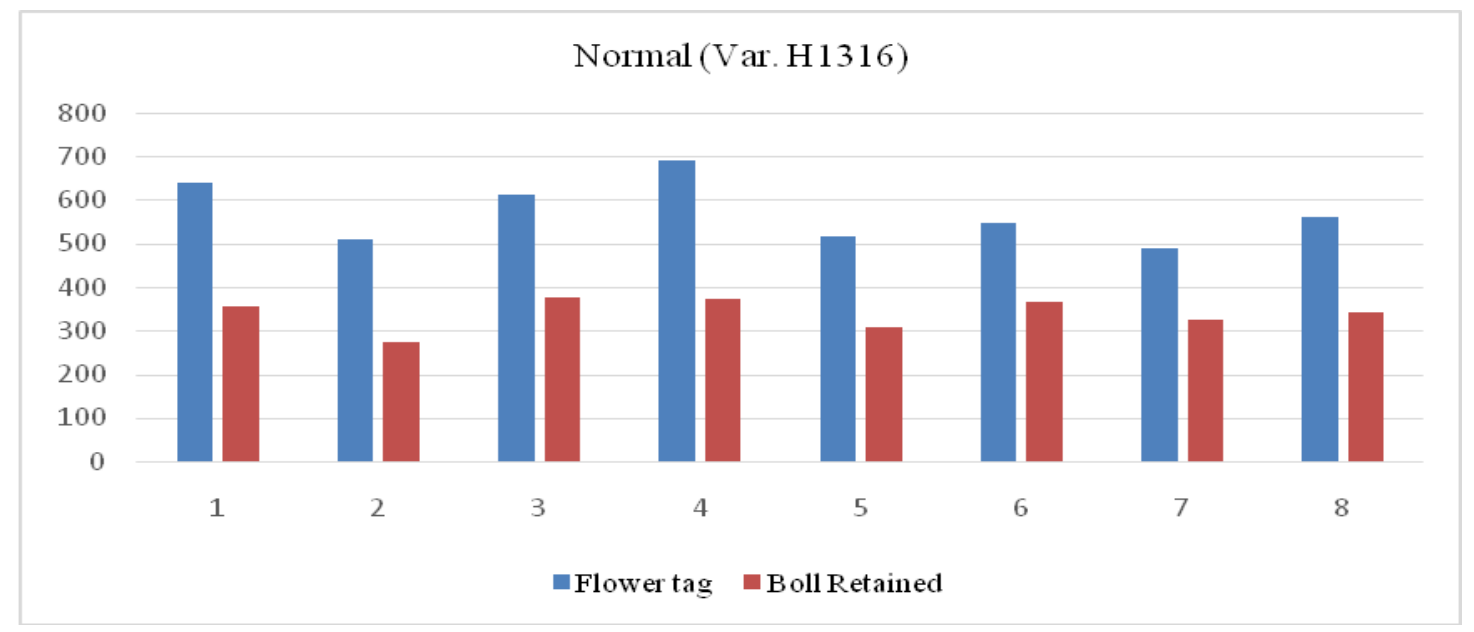

Fig.7 Boll retention in H1098 - I in late sown condition

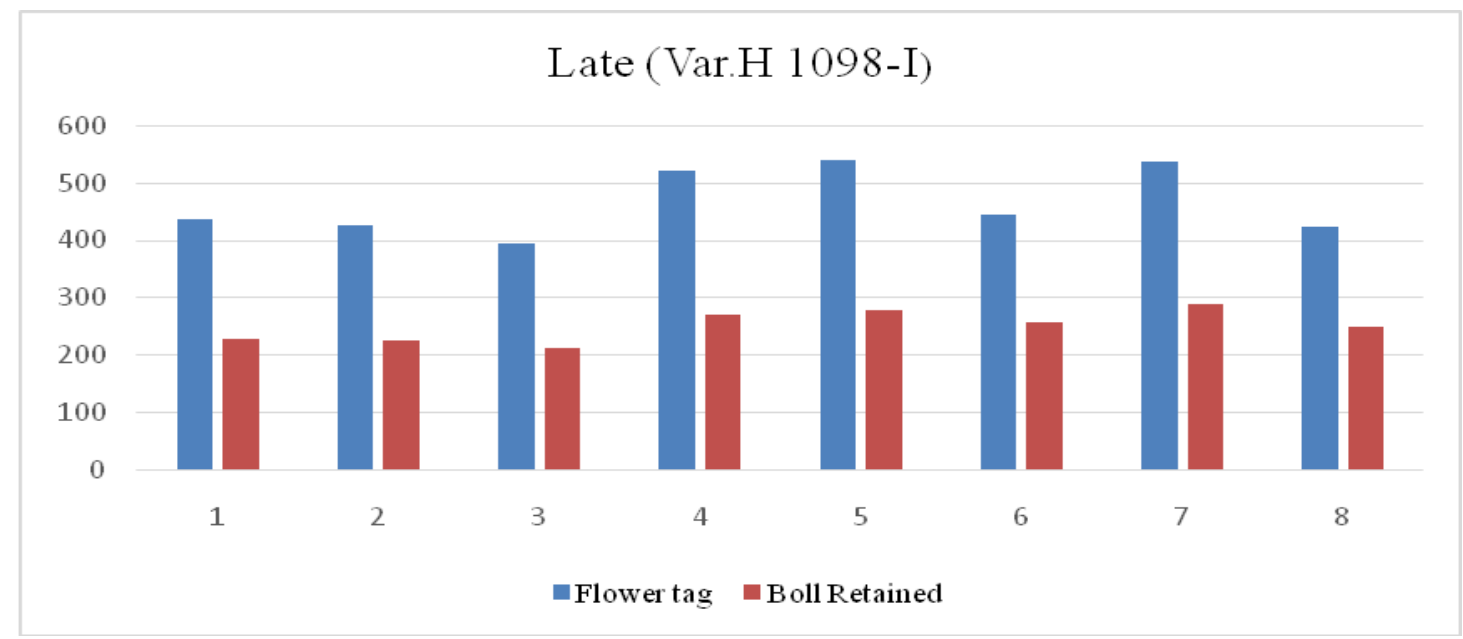


Fig.8 Boll retention in $\mathrm{H} 1300$ in late sown condition

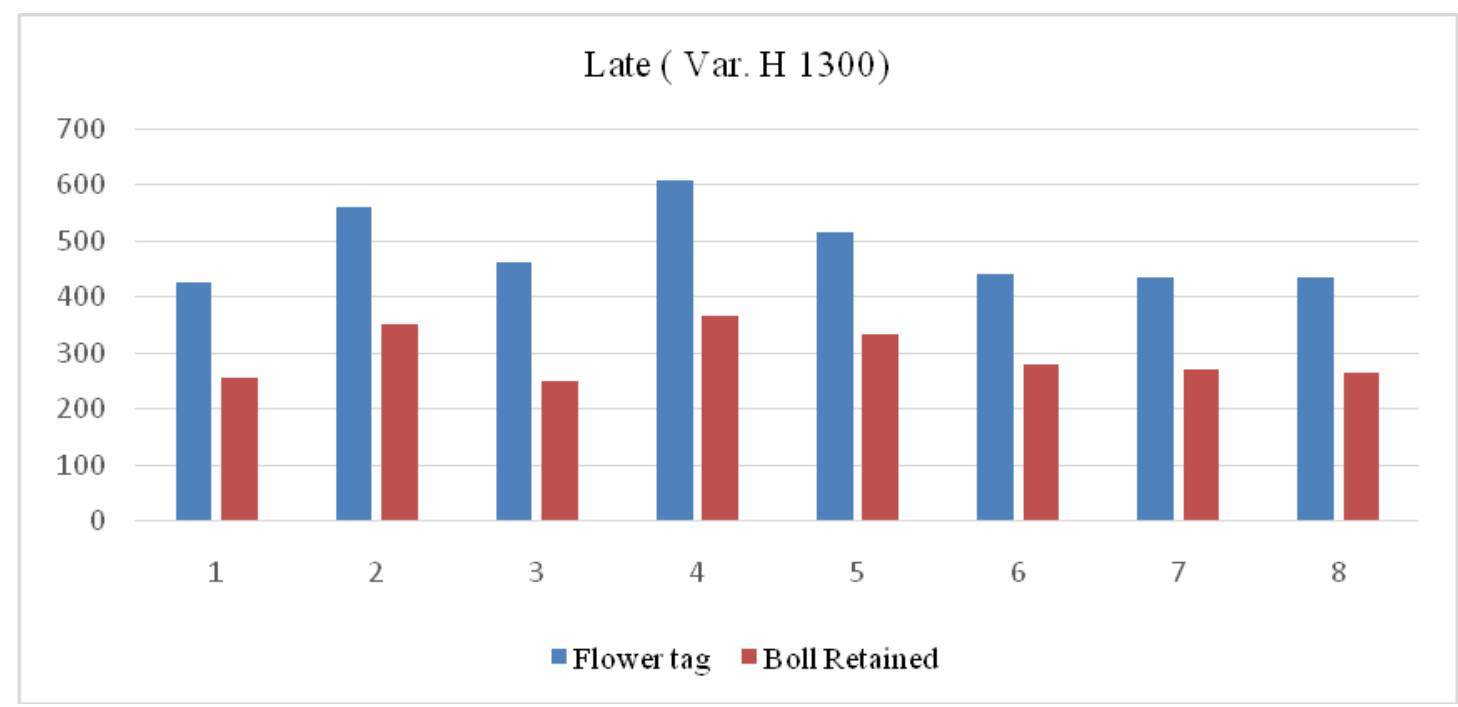

Fig.9 Boll retention in $\mathrm{H} 1 \mathrm{H} 1316$ in late sown condition

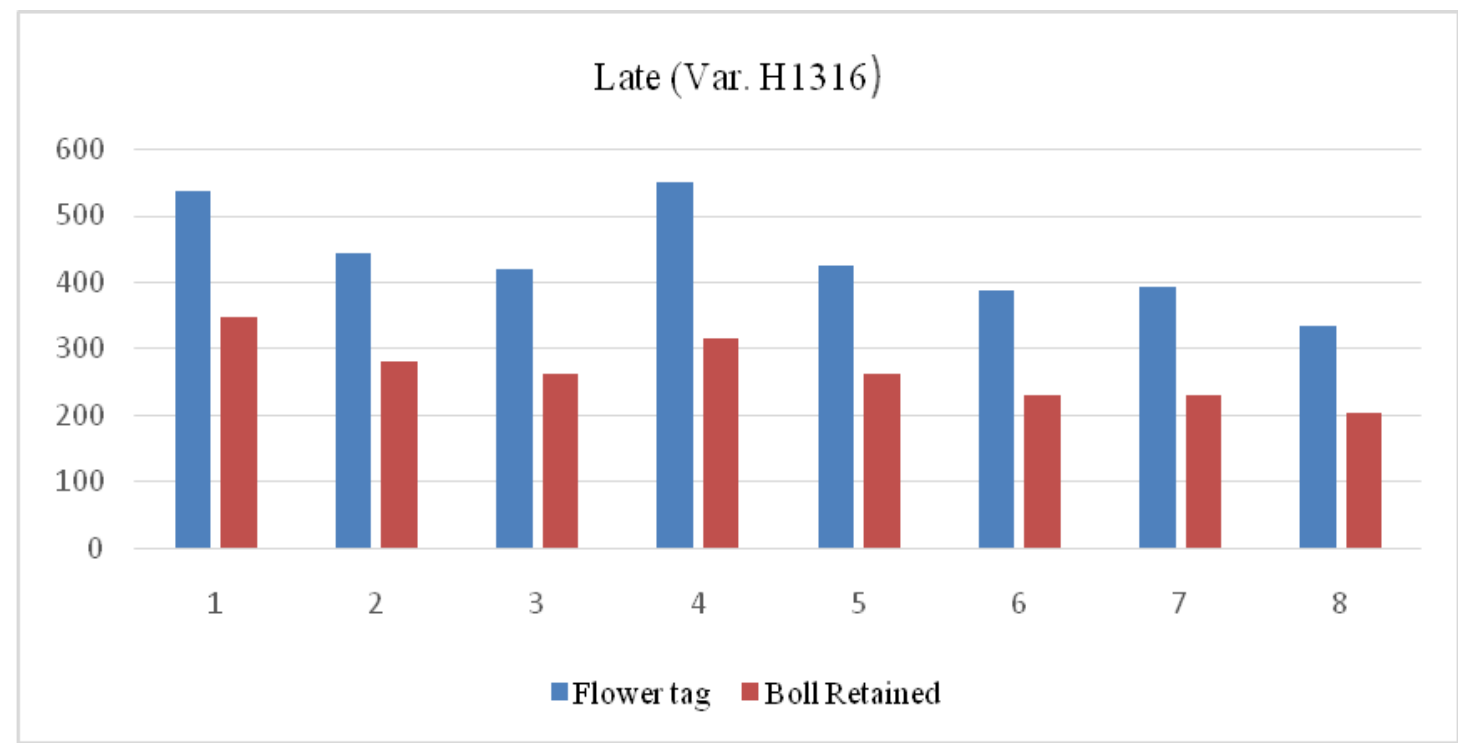

In early sown condition total flower tagged $(\mathrm{H}$ 1098-I, H 1300 and H 1316) were (6720, 7078 and 6887) 20685, total boll retained were (3665, 4485 and 4074) 12224 with 59.1 $\%$ boll retention.

In normal sown condition there were 8 tagging weeks from $8^{\text {th }}$ July to $26^{\text {th }}$ of August. In normal sown condition total flower tagged (H 1098-I, H 1300 and H 1316) were (4435, 5380 and 5229) 15044, total boll retained were $(2456,2628$ and 2717$) 7801$ with $51.9 \%$ boll retention. In late sown condition there were 8 tagging weeks from $8^{\text {th }}$ July to $26^{\text {th }}$ of August. In late sown condition total flower tagged (H 1098-I, H 1300 and H 1316) were (3720, 3876 and 3481) 11077, total boll retained were (2001, 2373 and 2126) 6500 with $58.7 \%$.boll retention. However, when the data was pooled for all the three conditions the boll retention was $56.6 \%$ (Table 8). 
In early sown condition total flower tagged were 20685, total boll retained was 12224 and $\%$ boll retention was 59.1. In normal sown condition total flower tagged were 15044, total boll retained was 7801 and $\%$ boll retention was 51.9. In late sown condition total flower tagged were 11077, total boll retained was 6500 and \% boll retention was 58.7. Total flower tagged in all three sown conditions were 46806 , total boll retained were 26525 and $\%$ boll retention was $56.6 \%$.

In early sown condition flower tagged were 20685, bolls retained were 12224 and \% boll retention was 59.1. In normal sown condition flower tagged were 15044, picked bolls were 7801 and \% boll setting was 51.9. In late sown condition flower tagged were 11077, bolls retained were 6500 and \% boll retention was 58.7. Total flower tagged in all three sown conditions were 46806 , bolls retained were 26525 and \% boll retention was $56.6 \%$. Thus per cent boll retention in early sown condition was higher $(59.1 \%)$ as compared to normal (51.9\%) and late (56.6\%) sown conditions. It was because of high temperature during late sown condition. Similar results were reported by Sawan et al., 2004. Temperature magnitude was the important climatic factor affecting flower and boll production as its correlation (negative) values were significant. In this respect, Warner and Burke (1993) indicated that the cool-night inhibition of cotton growth correlates with biochemical limitation on starch mobilization in source leaves, which results in a secondary inhibition of photosynthesis, even under optimal temperatures during the day. The second most important climatic factor in our study was minimum humidity, which was positively and highly correlated affecting number of flowers or harvested bolls in cotton. This means that high humidity rates during the 2 weeks preceding or succeeding initiation of flowers opening tended to significantly increased flower and boll production. The third most important climatic factor in our study was sunshine duration, which showed a significant positive relationship with boll production. The relationship between sunshine duration and boll retention might be due to the fact that species of the genus Gossypium are known to be short-day plants (Hearn and Constable 1984). Thus, an increase in sunshine duration above thresh hold resulted in decreased flower and boll production. Climatological factors and the boll load from the first fruiting cycle were evaluated as primary causes for low boll retention by cotton. The early prediction of possible adverse effects of climatic factors could help in minimizing their deleterious effects on cotton production through applying appropriate production practices, which would reduce any possible production shortage.

Sowing date, year and interaction (sowing date $\times$ year) all significantly affected the yield. Thus, sowing period was an important factor affecting the yield, biomass and reproductive duration and hence minimized the impact of temperature and duration of the reproductive growth stage. With climate change, an earlier planting date might be an efficient method of increasing yield in the future.

\section{References}

Hearn, A. B. and Constable, G. A. (1984). The Physiology of Tropical Food Crops. Chapter 14: 495-527.

Mahmood-ul-Hassan, N., Muhammad, Z. I., Muhammad, M Taj, Muhammad, I. and Saghir, A. (2003). Effect of Different Sowing Dates on Cotton (Gossypium hirsutum L.) Varieties. Asian J. Plant Sci. 2 (6): 461-463.

Malagouda, P. Khadi, B.M. Basamma, K. and I.S. Katageri (2014).Genetic Variability and Correlation Analysis for Fibre 
Quality Traits in Diploid Cotton (Gossypium spp). J. Agric. \& Environ. Sci. 14 (5): 392-395.

Sawan, Z. M., Hanna, L. I. and McCuistion, W. L. (2004).Effects of climatic factors prevailing prior to flowering or subsequent to boll setting on flower and boll production and retention of cotton in Egypt. J. Agro. \& Crop Sci. 190: 287-297.

Warner, D. A., and Burke, J. J. (1993). Cool night temperature after leaf starch and photosystem II. Chlorophyll fluorescence in cotton. Agron. J. 85: 836-840.

\section{How to cite this article:}

Pinki, S.S. Siwach, R.S. Sangwan, Sombir Singh, V.S. Mor, Shiwani Mandhania, Sunayana and Neha Rohila. 2018. Boll Retention (\%) under Different Environments/Sowing Conditions in Upland Cotton (Gossypium hirsutum L.). Int.J.Curr.Microbiol.App.Sci. 7(05): 877-886. doi: https://doi.org/10.20546/ijcmas.2018.705.107 